\title{
Alister McGrath* \\ Natürliche Theologie: Ein Plädoyer für eine neue Definition und Bedeutungserweiterung
}

https://doi.org/10.1515/nzsth-2017-0016

Zusammenfassung: Natürliche Theologie ist bis heute sowohl auf dem Gebiet der Systematischen Theologie als auch der Religionsphilosophie eines der interessantesten Themengebiete. Dieser Aufsatz erläutert, wie wichtig es ist, die Bedeutung von Natürlicher Theologie zu überdenken. Allgemein wird von einem Verständnis ausgegangen, das den Beweis der Rationalität des christlichen Glaubens im Allgemeinen oder ein plausibles Argument für die Existenz Gottes im Besonderen bedeutet, ohne sich dabei auf religiöse Annahmen zu berufen oder diese vorauszusetzen. Durch eine genealogische Analyse zeigt dieser Aufsatz, dass der Begriff „Natürliche Theologie“ in der christlichen Tradition an einigen Stellen in einer deutlich unterschiedlichen Bedeutung verwendet worden ist, und dass der in der Gegenwart vorherrschende Sinn die kulturellen und intellektuellen Belange des siebzehnten und frühen achtzehnten Jahrhunderts widerspiegelt. Sechs einzelne (wenn auch verwandte) Verständnisweisen der Natürlichen Theologie werden herausgearbeitet, von der jedoch keine als selbstverständlich richtig oder als normatives Verständnis des Konzepts verstanden werden kann. Abschließend wird festgehalten, welche positiven Folgen es haben könnte, wenn ein breiteres oder tieferes Verständnis der Natürlichen Theologie akzeptiert werden würde.

Schlüsselwörter: Natürliche Theologie, Rationalität, Existenz Gottes

Summary: Natural theology remains one of the most interesting fields of discussion within the fields of both systematic theology and the philosophy of religion. This article suggests that there is a need to review what is understood by the notion of natural theology, which is generally assumed to mean the demonstration of the rationality of the Christian faith in general, or a plausible argument for the existence of God in particular, without appealing to or presupposing any religious presuppositions. On the basis of a genealogical analysis, this article suggests that the term natural theology has been used in a number of quite different senses within the Christian tradition, and that the presently dominant

*Korrespondenzautor: Alister McGrath, Oxford University, Harris Manchester College, Oxford, OX1 3TD, United Kingdom, Faculty of Theology and Religion,

E-Mail: alister.mcgrath@theology.ox.ac.uk 
sense of the term reflects the cultural and intellectual concerns of the seventeenth and early eighteenth centuries. Six distinct (though often related) understandings of natural theology are identified, none of which can be considered to be a selfevidentally correct or normative understanding of the concept. Finally, some possible outcomes of accepting a wider and deeper notion of natural theology are noted.

Keywords: natural theology, rationality, existence of God

„Es ist die Aufgabe unserer theologischen Generation, sich zur rechten theologia naturalis zurückzufinden."1 Mit diesen Worten, aus dem Jahr 1934, legt Emil Brunner seine Überzeugung dar, dass man eine Form der Natürlichen Theologie wiedererschaffen und erneut etablieren müsse, die wieder mit den kulturellen Belangen seiner Zeit in Verbindung zu bringen sei. Den Kontext von Brunners Vorschlag bietet die große Debatte des Jahres 1934 zwischen Brunner und Karl Barth über Natur und Anmut, die vor dem Hintergrund des Aufstiegs des Nationalsozialismus stattfand, und die, (wenn auch nur als ein Element davon), eine Auseinandersetzung mit der Natürlichen Theologie umfasste. Die Qualität dieser Debatte wurde jedoch sowohl durch polemische Überlegungen als auch Barths offensichtlichem Verständnismangel für Brunners Ansatz und Absichten vermindert. $^{2}$

Die Aufgabe, ein kohärentes und theologisch legitimes Konzept der Natürlichen Theologie vorzulegen, ist dennoch insofern weiterhin essentiell für die christliche Lehre, Apologetik und Spiritualität, als sie einige der wichtigsten religiösen und kulturellen Fragen dieses Jahrhunderts aufwirft. Deuten zum Beispiel die Wunder der natürlichen Welt auf eine tiefgreifende Ordnung der Dinge, auch wenn diese nur zum Teil erhascht werden kann, statt vollkommen erfahren? Leitet uns die Natur - um die Bildsprache Dante Alighieris zu bemühen - durch eine „verborgene Höhlung zur lichten Erde”? ${ }^{3}$ Viele, die in den Naturwissenschaften arbeiten, sehen, dass diese Disziplinen tiefe Fragen über die Bedeutung des

1 Emil Brunner, “Natur und Gnade,” in Ein offenes Wort: Vorträge und Aufsätze 1917-1962. 2 Bd. (Zürich: Theologischer Verlag, 1981), Bd. 1, 374-5.

2 Vgl. Klaus-Peter BlaSER, "Communiquer l'incommunicable révélation: Le conflit Barth-Brunner revisité à la lumière de leur correspondance,” Etudes Théologiques et Religieuses 78 (2003), 59-67; Gerhard SAUTER, "Theologisch miteinander Streiten: Karl Barths Auseinandersetzung mit Emil Brunner,” in Karl Barth in Deutschland (1921-1935): Aufbruch - Klärung - Widerstand, Hrsg. Michael BeintKer, Christian Link und Michael Trowitzsch (Zürich: Theologischer Verlag, 2005), 267-84; Alister E. McGrath, Emil Brunner: A Reappraisal (Oxford: Wiley-Blackwell, 2014), 90132.

3 Dante, Inferno, 34, 133-5. [Übersetzung Hermann Gmelin]. 
Lebens aufwerfen, die auf der Grundlage der wissenschaftlichen Methodik nicht beantwortet werden können. Dies eröffnet neue Möglichkeiten des Dialogs innerhalb einer allgemeinen Kategorie der „Natürlichen Theologie”. ${ }^{4}$

Es ergeben sich jedoch bedeutende Probleme bei jedwedem Versuch, über eine angemessene Interpretation und Umsetzung der Natürlichen Theologie nachzudenken, und dabei weit über die beschränkte, (und nicht unbedingt erhellende), Debatte von 1934 zwischen Barth und Brunner hinaus zu kommen. ${ }^{5}$ Das Konzept der Natürlichen Theologie wird sowohl in der Religionsphilosophie als auch in der systematischen Theologie umfassend besprochen; doch diese Ausführungen werden oft von einem vorgefassten Verständnis, was Natürliche Theologie sein soll, beeinflusst, wenn nicht sogar vorbestimmt. Viele nehmen mittlerweile an, dass die korrekte und unanfechtbare Bedeutung des Begriffs „Natürliche Theologie” die Unternehmung sei, religiösen Glauben zu stärken, indem von Prämissen ausgegangen wird, die weder religiösem Glauben entsprechen noch diesen voraussetzen. ${ }^{6}$ Eine unkritische Akzeptanz dieser „offiziellen” Sichtweise führt dazu, dass einige Theolog^innen „Natürliche Theologie“ als unorthodox, möglicherweise sogar häretisch betrachten. ${ }^{7}$ Doch diese spezielle Auffassung von Natürlicher Theologie entstand erst während der Englischen Aufklärung und erlangte im achtzehnten Jahrhundert die kulturelle Vorherrschaft.

Wir müssen uns also fragen, warum dieser Missstand so fortbestehen darf? Er mag die Sichtweise des theologischen Establishments widerspiegeln, besonders auf dem Gebiet der Religionsphilosophie. Doch diese Auffassung von Natürlicher Theologie ist nur eine Möglichkeit, ein Nachzügler, und noch dazu eine relativ junge Variante. Die theologische Tradition muss eindeutig danach befragt werden, warum man dieser speziellen Auffassung von Natürlicher Theologie erlaubt hat, eine solche Vormachtstellung einzunehmen, sogar in einer post-aufgeklärten Welt. Warum soll die Natürliche Theologie von modernistischen Rahmenbedingungen eingeengt und dadurch auf einen Beweis für die Rationalität des Glaubens an Gott eingeschränkt werden? Was ist mit ihrem Bezug auf Schönheit und

4 Vgl. FernandoVidal and Bernard Kleeberg, "Knowledge, Belief, and the Impulse to Natural Theology,” Science in Context 20 (2007), 381-400; Johannes Maria Stenke, John Polkinghorne: Konzonanz von Naturwissenschaft und Theologie (Göttingen: Vandenhoeck \& Ruprecht), 2006.

5 Vgl. z.B. Evangelia G. DAfNI, "Natürliche Theologie im Lichte des hebräischen und griechischen Alten Testaments," in Theologische Zeitschrift 57 (2001), 295-310; Christoph Koск, Natürliche Theologie: Ein evangelischer Streitbegriff (Neukirchen-Vluyn: Neukirchener, 2001); Henning Tegtmeyer, Gott, Geist, Vernunft: Prinzipien und Probleme der natürlichen Theologie (Tübingen: Mohr Siebeck 2013).

6 Vgl. William P. Alston, Perceiving God: The Epistemology of Religious Experience (Ithaca/NY: Cornell University Press, 1991), 289.

7 Коск, Natürliche Theologie, 391-412. 
Vorstellungskraft? Das Vermächtnis der modernistischen Unterdrückung der Imagination sucht immer noch die Praxis der systematischen Theologie heim und verarmt unsere Konzeption der Natürlichen Theologie.

Die Problematik Brunners bleibt auch für die nachfolgenden Generationen von Theolog*innen bestehen, besonders im Kontext neuerer Diskussionen über Rationalität und Integrität des Glaubens und dessen Beziehung zu anderen Disziplinen des menschlichen Forschens, insbesondere der Naturwissenschaften. Dieser Aufsatz dient als Aufforderung dazu, unseren Weg zu einer solchen Natürlichen Theologie zurück $\mathrm{zu}$ finden, in dem Bewusstsein, dass die christliche Geschichte reich an Ansätzen ist, die durch die vorherrschenden theologischen Stimmen und Institutionen verdrängt oder unterdrückt wurden, die jedoch der heutigen theologischen Gemeinschaft gute Dienste leisten würden. Die wachsende Anerkennung der Bedeutung von „Natur“, „Wissenschaft“ und „Religion“ als soziale Konstrukte, besonders in der Frühen Neuzeit, ${ }^{8}$ weist darauf hin, dass es keine vorherbestimmte, wesentliche Form von Natur oder Natürlichen Theologie gibt; sondern dass beides vielmehr einer kulturellen Revision und ideologischen Rekonstruktion offen steht, die eine soziale und kulturelle Verortung der jeweiligen Ausführung vornimmt. Somit stellt sich die Frage, wie wir eine Vorstellung von Natürlicher Theologie konstruieren können, die nicht durch die Normen und Vorannahmen der vorherrschenden Gegebenheiten längst vergangener Zeiten bestimmt ist.

Es ist wenig sinnvoll über den Begriff der „Natürlichen Theologie“ zu diskutieren, während man von den bekannten Definitionen ausgeht, so als ob ihre Bedeutung selbsterklärend wäre. Der erste Schritt, um ein brauchbares Verständnis von Natürlicher Theologie zu entwickeln, ist es, sich ihrer Geschichte genealogisch zu nähern und dadurch herauszufinden, auf welche Weise die Idee verstanden worden ist. ${ }^{9}$ Konzepte haben eine Geschichte und aus diesem Grund haben sie eine Genealogie, die zurückverfolgt werden muss, um unser Verständnis dieser Konzepte und der Denkweisen, die sie geformt haben, zu vertiefen. Es ist inakzeptabel, eine zeitgenössische Definition von „Natürlicher Theologie“ anzubieten, die innerhalb einer speziellen Diskursgemeinschaft akzeptiert wird, als ob dies die Angelegenheit beenden würde. Die Genealogien von Kernkonzepten $\mathrm{zu}$ untersuchen, ist eine der effektivsten, (wenn auch nicht unproblematischen) Wege, unterdrückte oder verdrängte Bedeutungen wieder aufzufinden, die

8 Vgl. besonders Peter Harrison, The Territories of Science and Religion(Chicago: University of Chicago Press, 2015), 1-19.

9 Eine erhellende Anwendung dieses Ansatzes findet man bei Vincent RzepкA, Die Ordnung der Transparenz: Jeremy Bentham und die Genealogie einer demokratischen Norm (Berlin: LIT, 2013). 
trotzdem durchaus lebendige intellektuelle Räume für eine zeitgenössische theologische Debatte bieten.

Der lateinische Begriff theologia naturalis - den man sowohl mit „natürliche Theologie“ als auch als „Naturtheologie“ oder „Theologie der Natur“ übersetzen kann - wurde in der antiken Welt geprägt, um allgemein eine Denkweise zu beschreiben, die von der natürlichen Welt in die Welt der Götter vordrang. ${ }^{10}$ In der römischen Religion war die theologia naturalis eine von drei Säulen der Religion, als Ergänzung zur theologia civilis und theologia mythica. ${ }^{11}$ Obwohl er seine Abhandlung auf Latein verfasste, verwendete der Philosoph Varro drei griechische Adjektive (mythicon, politicon und physicon), um die tria genera theologiae zuzuordnen. Dies legt die Vermutung nahe, dass diese Kategorisierung aus der Philosophie (und Sprache) des hellenistischen Zeitalters übernommen wurde. ${ }^{12}$

Der Ausdruck theologia naturalis wurde von Einigen der frühchristlichen Theolg^innen aufgegriffen, wie Augustinus von Hippo, ${ }^{13}$ der es gerne als abwertende Bezeichnung für die minderwertigen Theologien heidnischer Philosophen verwendete. Der Begriff fand jedoch in der westlichen theologischen Tradition keine weitreichende Akzeptanz. Er wurde während der patristischen Zeit und des Mittelalters wenig gebraucht und verbreitete sich erst wieder im sechzehnten Jahrhundert. Als Konsequenz verwenden Historiker`innen und Theolog^innen den Begriff „Natürliche Theologie“ normalerweise in retrospektiver Weise - zum Beispiel, um über die „natürliche Theologie Thomas von Aquins“ zu sprechen (und damit zu umschreiben, was Thomas von Aquin heute als „Natürliche Theologie“ bezeichnen würde, obwohl dies nicht die Terminologie ist, die Thomas selbst bevorzugte).

Es ist relativ einfach aufzuzeigen, dass die allgemeine Akzeptanz und weitreichende Verbreitung des Begriffs theologia naturalis in der westlichen theologischen Tradition den Einfluss von Sebondes Liber naturae sive creaturarum widerspiegelt. Es herrscht allerdings einige Verwirrung bezüglich des ursprünglichen Titels von Sebondes Abhandlung. Die Manuskripte, die in führenden europäischen Bibliotheken zu finden sind, weisen recht unterschiedliche Titel auf: Liber Naturae sive Creaturarum (Paris), Scientia Libri creaturarum seu Naturae et de

10 Hermann Dembowsкi, “Natürliche Theologie -Theologie der Natur,"Evangelische Theologie 45 (1985), 224-48; Markus Enders, Natürliche Theologie im Denken der Griechen (Frankfurt am Main: Josef Knecht, 2000), 47-73.

11 Vgl. Jörg RứpKe, Die Religion der Römer: Eine Einführung (München: C. H. Beck, 2001), 121-5.

12 Vgl. Augustine, de Civitate Dei, VI.v.12.

13 Vgl. Albrecht Dihle, "Die Theologia Tripertita bei Augustin," in Geschichte-Tradition-Reflexion: Festschrift für Martin Hengel zum 70. Geburtstag, Hrsg. Hubert CANCIK (Tübingen: Mohr Siebeck, 1996), 183-202. 
Homine (Toulouse) und Liber Creaturarum sive de Homine (Clermond-Ferrand). Der entscheidende Untertitel Theologia naturalis wurde erst in der zweiten Auflage (1485) von den Herausgeber^innen hinzugefügt, ein halbes Jahrhundert nach Sebondes Tod. Vor allem aufgrund des Einflusses des Renaissance-Philosophen Michel de Montaigne (1533-92), der eine französische Übersetzung von Sebondes Werk mit dem Titel $L a$ théologie naturelle veröffentlicht hatte, trat der Untertitel schließlich an die Stelle des ursprünglichen Titels. ${ }^{14}$ Montaignes Übersetzung war so einflussreich, dass sich dieser Titel nicht nur weiter verbreitete, sondern spätere Leser auch davon ausgingen, dies sei der Originaltitel von Sebondes Werk.

Wie die meisten Denker der Renaissance definierte Sebonde Natürliche Theologie nicht in polemischen Begrifflichkeiten, - zum Beispiel im Gegensatz zur göttlichen Offenbarung -, sondern betrachtete stattdessen das „Buch der Geschöpfe" selbst als eine Art der Offenbarung durch die Gott entschied, menschlichen Beobachtern (sowohl kognitives als auch affektives) Wissen zu übermitteln. Sebondes Herangehensweise hat es erschwert, eine klare Unterscheidung zwischen natürlicher Theologie und Naturphilosophie vorzunehmen. ${ }^{15}$ Auch deutet Sebonde die theologia naturalis nicht in bloß kognitiven Begrifflichkeiten; er versteht darunter eindeutig auch eine affektive Auseinandersetzung mit oder Annäherung an die natürliche Ordnung. Sebondes Abhandlung ist, obwohl sie Abschnitte beinhaltet, die sich mit dogmatischer Theologie auseinandersetzen, ebenso ein Werk der Spiritualität, wie auch eines der Theologie. ${ }^{16}$ Wo moderne Vorstellungen der natürlichen Theologie sich mit der Herausforderung des Atheismus auseinandersetzen, war Sebonde mehr daran interessiert, seinen Leser`innen dabei zu helfen, mehr von der Schönheit und Pracht Gottes zu begreifen, die in und durch die natürliche Ordnung zu erkennen sind.

Heute nehmen natürlich viele an, dass sich die Natürliche Theologie auf einen Weg bezieht, die Existenz Gottes zu beweisen, ohne irgendwelche expliziten religiösen Vorannahmen einnehmen zu müssen, und behaupten dadurch, dass Sebonde eine „idiosynkratische“ oder „unorthodoxe“ Auffassung der Natürlichen Theologie vertritt, ${ }^{17}$ die außerhalb einer informierten Haltung zu diesem

14 Vgl. Michel Simonin, "La préhistoire de l'Apologie de Raimond Sebond," in Montaigne, Apologie de Raimond Sebond: De la theologia à la théologie, Hrsg. Claude Blum (Paris: Honoré Champion, 1990), 85-116.

15 Vgl. Ann Blair, "Mosaic Physics and the Search for a Pious Natural Philosophy in the Late Renaissance," in Isis 91, Nr. 1 (2000), 32-58.

16 Vgl. Jean Balsamo, "Un gentilhomme et sa théologie," in Dieu à nostre commerce et société: Montaigne et la théologie, Hrsg. Philippe Desan (Genf: Droz, 2008), 105-26.

17 Vgl. Ann Hartle, Michel de Montaigne: Accidental Philosopher (Cambridge: Cambridge University Press, 2003), 141. 
Thema liegt. Dieses Urteil ist jedoch verfehlt. Es resultiert daraus, dass ein normatives Verständnis von Natürlicher Theologie zugelassen wird, das in einem späteren kulturellen Kontext vorherrschend wurde, und damit festzulegen, wie die Idee in früheren Zeiten hätte verstanden werden sollen. Ein realistischerer Vorschlag wäre es, zuzulassen, dass Sebondes breiteres Verständnis von Natürlicher Theologie aufgrund historischer Priorität als normativ gesehen wird und damit die späteren Ausführungen der Idee als Beschränkung oder den Umfang beschneidend darzustellen.

Historisch betrachtet bezeichnet der Ausdruck „Natürliche Theologie“ tatsächlich eine Vielfalt an Möglichkeiten, ohne offensichtliche Gründe einer davon aus einer unabhängigen Perspektive gesehen den Vorzug zu geben. Das wirft die Frage auf, ob die Bedeutung grundsätzlich inkohärent ist, oder ob es eine übergreifende Bedeutung von natürlicher Theologie gibt, die in der Lage ist, ihre verschiedenen Ausprägungen innerhalb eines breiteren intellektuellen Kontexts $\mathrm{zu}$ vereinen. Im Folgenden werden die sechs Hauptausrichtungen - oder Verständnisarten - der Natürlichen Theologie erörtert, die innerhalb der westlichen Theologie festzumachen sind.

\section{Natürliche Theologie: Sechs Herangehensweisen}

Innerhalb der westlichen Tradition lassen sich sechs Hauptbedeutungen des Begriffs der natürlichen Theologie identifizieren. ${ }^{18}$ Keine davon kann als unumstößlich angesehen werden und jede einzelne könnte über den relativ engen Bedeutungsumfang, der im Folgenden skizziert wird, hinaus entwickelt werden. Jede kann als Konstruktion oder Interpretation eines umfänglicheren und tiefer reichenden zugrunde liegenden Konzepts gesehen werden, das die Bedürfnisse oder Möglichkeiten des jeweiligen Kontextes, in das es eingebettet ist, widerspiegelt.

1. Natürliche Theologie ist der Zweig der Philosophie, der untersucht, was der menschliche Verstand uns ohne die Hilfe der Offenbarung über Gott sagen kann. Sie wird hier als Versuch verstanden, die Existenz Gottes oder seinen Charakter aufzuzeigen, ohne auf die göttliche Offenbarung zurückzugreifen. ${ }^{19}$ Für viele ist

18 Für weitere Ausführungen zur konzeptuellen Vielfältigkeit der „Natürlichen Theologie“ vgl. David Fergusson, "Types of Natural Theology," in The Evolution of Rationality: Interdisciplinary Essays in Honor of J. Wentzel van Huyssteen, Hrsg. F. LeRon Schults (Grand Rapids, MI: Eerdmans, 2007), 380-93.

19 Vgl. Paul Clavier, Qu'est-ce que la théologie naturelle? (Paris: Vrin, 2004); Christian WeideMANN, Die Unverzichtbarkeit natürlicher Theologie (München: Karl Alber, 2007), 44-7. 
dies in der Zwischenzeit zur Standardbedeutung von Natürlicher Theologie geworden. Diese Art des Verständnisses hängt weder von irgendwelchen speziellen christlichen Ideen $\mathrm{ab}$, noch drückt sie solche aus, und übt dadurch innerhalb eines säkularen kulturellen Kontexts einen starken apologetischen Reiz aus. Sie beruft sich nicht auf die natürliche Welt selbst, sondern vielmehr auf a prioriIdeen, die als „natürlich“ gesehen werden könnten und erforscht deren theistische Folgerungen. Natürliche Theologie umfasst also eine Theologie, die dem menschlichen Bewusstsein „natürlich“ entspringt - wie zum Beispiel Anselm von Canterburys ontologisches Argument. ${ }^{20}$

2. Natürliche Theologie ist ein Beweis oder eine Bestätigung für die Existenz Gottes auf der Grundlage der Regelmäßigkeit und Komplexität der natürlichen Welt. Diese spezielle Formulierung der Natürlichen Theologie scheint sich hauptsächlich aus apologetischen Gründen während der Frühen Neuzeit in protestantischen Kontexten entwickelt zu haben. Sie wird oft als ,Physikotheologie,21 bezeichnet, da sie sich a posteriori auf ein Vermögen beruft die Regelmäßigkeit der Natur wahrzunehmen, aus der die göttliche Existenz folgt,oder die sie impliziert. Dadurch dass sie sich auf die allgemeine Welt der Natur bezieht, vermeidet diese Verständnisart den „Skandal der Besonderheit“, der sich daraus ergibt, dass der Modernismus darauf besteht, das Wissen um das Göttliche müsse universal zugänglich und nicht historisch oder kulturell spezifisch sein. ${ }^{22}$

Innerhalb des Katholizismus wurde dieses Verständnis von Natürlicher Theologie im neunzehnten Jahrhundert zunehmend bedeutsamer, als der Säkularismus in Westeuropa zu einem drängenden Problem wurde. Diese Strategie ist in der Erklärung des Ersten Vatikanischen Konzils erkennbar, wonach Gott mit Gewissheit aus der Betrachtung der Schöpfung im natürlichen Licht des menschlichen Verstandes erkannt werden könne. ${ }^{23}$ Obwohl offensichtliche Parallelen

20 Vgl. Peter Hinst, “A Logical Analysis of the Main Argument in Chapter 2 of the Proslogion by Anselm of Canterbury," in Philosophiegeschichte und Logische Analyse 17 (2014), 22-44.

21 Vgl. Peter Harrison, "Physico-Theology and the Mixed Sciences: The Role of Theology in Early Modern Natural Philosophy,” in The Science of Nature in the Seventeenth Century Hrsg. Peter Anstey and John Schuster (Dordrecht: Springer, 2005),165-83.

22 Vgl. Owen Anderson, "The Presuppositions of Religious Pluralism and the Need for Natural Theology," in Sophia 47 (2008), 201-22.

23 Vgl. "Si quis dixerit, Deum unum et verum, creatorem et Dominum nostrum, per ea, quae facta sunt, naturali rationis humanae lumine certo cognosci non posse: anathema sit," Heinrich Denzinger, Enchiridion Symbolorum Definitionum et Declarationum de Rebus Fidei et Morum. 39. Aufl. (Freiburg-im-Briesgau: Herder, 2001), \#3043. Zum historischen Kontext dieser Aussage vgl. Daniele Menozzi, “Antimodernismo, secolarizzazione e cristianità,” in Il modernismo tra cristianità e secolarizzazione, Hrsg. Alfonso Botti and Rocco Cerrato (Urbino: Quattro Venti, 2000), 53-82. 
zwischen diesem Verständnis und dem ersten, wie oben ausgeführt, bestehen, birgt ihr Ausgangspunkt einen entscheidenden Unterschied: die erste erfolgt aus dem bloßen Verstand (wie in der aufklärerischen Umformulierung von Anselm von Canterburys ontologischem Argument), die zweite entsteht aus der Auseinandersetzung mit der Welt der Natur.

3. Natürliche Theologie ist das intellektuelle Ergebnis aus der natürlichen Tendenz des menschlichen Bewusstseins, Gott zu wollen, oder ihm geneigt zu sein. Dieses Verständnis beruft sich traditionellerweise auf das „natürliche Verlangen, Gott zu sehen“, das von Thomas von Aquin formuliert wurde, ${ }^{24}$ obwohl neuere Entwicklungen in der kognitiven Religionswissenschaft alternative Wege aufgezeigt haben, dieses Thema anzugehen. ${ }^{25}$ Andere Theolog*innen haben diese Idee auf wichtige Weise vorangebracht - vor allem Bernard Lonergan, der dieses Prinzip als immanente Tendenz des menschlichen Intellekts postulierte, die gleichzusetzen mit dem uneingeschränkten Verlangen sei, das Sein zu verstehen. ${ }^{26}$

4. Natürliche Theologie ist die Erforschung einer Analogie oder intellektuellen Resonanz zwischen dem menschlichen Erleben von Natur einerseits und dem christlichen Evangelium andererseits. Diese Verständnisart von Natürlicher Theologie beschränkt sich selbst oft darauf, die Möglichkeit einer Kohärenz oder Kongruenz zwischen den einzelnen Behauptungen des christlichen Glaubens und einem Weltwissen herzustellen, das aus anderen Disziplinen oder Lebensbereichen rührt. Natürliche Theologie artikuliert und erweitert demnach die Idee einer Isomorphie zwischen unserem Verstand und der Struktur der Realität. ${ }^{27}$ Varianten dieses Verständnisses lassen sich bei dem englischen Physiker und Theologen John Polkinghorne finden. ${ }^{28}$ In diese Kategorie können wir auch jene einbinden, für die die Natürliche Theologie die Rationalität eines bestehenden Glaubens

24 Vgl. Reinhard HÜTter, “Desiderium naturale visionis Dei - est autem duplex hominis beatitudo sive felicitas: Some Observations about Lawrence Feingold's and John Milbank's Recent Interventions in the Debate over the Natural Desire to See God," in Nova et Vetera 5 (2007), 81-132.

25 Vgl. Justin L. Barrett, Why Would Anyone Believe in God? (Lanham, MD: AltaMira Press), 2004.

26 Vgl. Brian Himes, "Lonergan's Position on the Natural Desire to See God and Aquinas' Metaphysical Theology of Creation and Participation,” in Heythrop Journal 54, Nr. 5 (2013), 76783.

27 Vgl. Margit Wasmaier-Sailer und Benedikt Paul Göcke, "Idealismus als Chance für die natürliche Theologie," in Idealismus und natürliche Theologie, Hrsg. Margit W ASMAIER-SAILER and Benedikt Paul GöcKE (Freiburg im Breisgau: Verlag Karl Alber, 2011), 9-28.

28 Vgl. Bernd Irlenborn, "Konsonanz von Theologie und Naturwissenschaft? Fundamentaltheologische Bemerkungen zum interdisziplinären Ansatz von John Polkinghorne,” Trierer Theologische Zeitung 113 (2004), 98-117. 
bestätigt, statt zunächst die Notwendigkeit dieses Glaubens aufzuzeigen, und die dadurch einen intellektuellen Rahmen bieten, um den Glauben der eigenen Tradition in Hinblick auf die Endgültigkeit in einer plausiblen und glaubensstärkenden Weise auszudrücken. ${ }^{29}$

5. Natürliche Theologie ist ein Versuch aufzuzeigen, dass „naturalistische“ Darstellungen der natürlichen Welt und die Errungenschaften der Naturwissenschaften immanent unzureichend sind, und dass eine theologische Herangehensweise notwendig ist, um eine umfassende und kohärente Interpretation der natürlichen Ordnung vornehmen zu können. Dieser Ansatz beinhaltet eine Auseinandersetzung mit dem kulturell scheinbar vorherrschenden Glauben, dass eine intellektuelle Reflektion über die Natur nun eher zu einer „natürlichen Atheologie“ als einer „natürlichen Theologie“ führen wird, ${ }^{30}$ wobei sich die fragwürdige Lückentheorie in der es einen Gott der Lücke oder Gott in der Lücke gibt, vermeiden lässt.

Dieser Herangehensweise an die Natürliche Theologie ist deshalb in gegenwärtigen Diskussionen über die metaphysischen Implikationen der Wissenschaft besonders wichtig, da sie die Idee, dass eine naturalistische Erklärung der Natur epistemologisch zu bevorzugen oder „neutral“ sei, infrage stellt. Indem sie offen mit ihren eigenen metaphysischen Annahmen oder narrativen Verpflichtungen umgeht, fordert sie vom Naturalismus, seine eigenen impliziten Grundfeste und Annahmen anzuerkennen. ${ }^{31}$ Genauer gesagt, besagt dieses Verständnis von Natürlicher Theologie, dass eine „wissenschaftliche“ Erklärung der Realität - die im Endeffekt die Realität auf eine bestimmte Schicht oder eine einzelne Perspektive beschränkt - schlichtweg unzulänglich ist, um das menschliche Erleben der Welt in all seinen Komplexitäten zu erklären.

6. Natürliche Theologie muss vor allem als eine „Theologie der Natur“ verstanden werden, also als ein speziell christliches Verständnis der natürlichen Welt, das die Kernannahmen des christlichen Glaubens widerspiegelt, und das wiederum den säkularen oder naturalistischen Erklärungsansätzen von Natur gegenübersteht. ${ }^{32}$ Der Gedankenablauf bewegt sich hier aus der christlichen

29 Vgl. Wesley J. Wildman, “Comparative Natural Theology,” in American Journal of Theology and Philosophy 27, Nr. 2-3 (2006), 173-90.

30 Vgl. Thomas Dixon, "Theology, Anti-Theology and Atheology: From Christian Passions to Secular Emotions,” in Modern Theology 15, Nr. 3 (1999), 297-330.

31 Vgl. Aku Visala, Naturalism, Theism and the Cognitive Study of Religion: Religion Explained? (Aldershot: Ashgate, 2011), 85-193.

32 Ein gutes Beispiel findet sich im Werk von Wolfhart Pannenberg. Vgl. Anja Lebrüche, Theologie der Natur: Wolfhart Pannenbergs Beitrag zum Dialog zwischen Theologie und Naturwissenschaft (Neukirchen-Vluyn: Neukirchener Verlag, 2011). Allgemein zum Thema vgl. Colin Gunton, 
Tradition heraus in Richtung Natur und nicht von der Natur ausgehend in Richtung des Glaubens, (so wie in der oben erläuterten zweiten Herangehensweise). Diese Art einer Theologie der Natur wird häufig vor allem in den Kontext der Schöpfungslehre eingebettet.

Mit Blick auf diese Pluralität von Interpretationsweisen, könnte man versucht sein zu folgern, dass das Konzept der „Natürlichen Theologie“ inkohärent ist, offen für eine so große Vielfalt an Interpretationen, dass sie nicht länger als legitimes oder bedeutsames Konzept Bestand hat. Einige haben auf diese Interpretationspluralität so reagiert, dass sie die intellektuelle und historische Hegemonie zu einer Art, Natürliche Theologie zu verstehen, erklären und darauf bestehen, dass rivalisierende Bedeutungskonstrukte fehlerhaft, möglicherweise sogar degeneriert seien. Das wird besonders deutlich bei denjenigen, die darauf bestehen, dass die einzig legitime Interpretation von Natürlicher Theologie eine apologetische Strategie ist, deren Sinn es ist, die Existenz Gottes auf rationaler Ebene zu beweisen oder bestätigen.

Meiner Meinung nach wird die Idee der Natürlichen Theologie durch die Vielfalt an Verständnisweisen weder inkohärent noch bedeutungslos. Ich habe an andere Stelle erläutert, dass die Vielfalt dieser sechs Modi des Diskurses deutlich macht, dass es einer größeren Vision der Natürlichen Theologie bedarf, die konzeptuellen Raum für jede dieser sechs Ausformungen birgt. ${ }^{33}$ Die großen Themen des christlichen Glaubens geben somit den Interpretationsrahmen vor, innerhalb dessen Natur tiefgreifend und bedeutsam betrachtet werden kann. Meiner Überzeugung nach bietet die intellektuelle Landkarte, die die christliche Tradition entwirft, ein imaginatives und kognitives Bezugssystem, das verschiedene Verständnisweisen von Natürlicher Theologie sowohl innerhalb einer umfassenderen kohärenten Vorstellung von Realität aufnehmen als auch verorten kann; vergleichbar mit der Art und Weise wie Einsteins relativistische Mechanik Newtons klassische Mechanik integrieren konnte, indem sie sie als Spezialfall einer größeren Idee sah oder diese Idee unter eingeschränkten Bedingungen.

Ich möchte jedoch betonen, dass dies nur ein positiver Vorschlag für weitere Überlegungen sein soll; das entscheidende Argument dieses Aufsatzes ist es, uns klarzumachen, dass wir nicht weiterhin eine unkritische und historisch falsch verortete Idee der Natürlichen Theologie akzeptieren können, die dazu dient, theistische Glaubensvorstellungen herzuleiten oder $\mathrm{zu}$ verteidigen, ohne auf theologische Annahmen zurückzugreifen. Dieser spezielle Ansatz entstand in

\footnotetext{
"The Trinity, Natural Theology, and a Theology of Nature," in The Trinity in a Pluralistic Age, Hrsg. Kevin VANHOOZER (Grand Rapids, MI: Eerdmans, 1997), 88-103.

33 Vgl. Alister E. McGrath, Re-Imagining Nature: The Promise of a Christian Natural Theology (Oxford: Wiley-Blackwell, 2016).
} 
Reaktion auf einen gleichermaßen speziellen kulturellen Kontext, der nun in der Vergangenheit liegt. Wir können es nicht länger zulassen, dass die Diskussion dieser wichtigen Idee in der Gegenwart weiterhin im Schatten der Vergangenheit geführt und bestimmt wird.

\section{Fazit}

Die spezielle Bedeutung von Natürlicher Theologie, in der sie Unterstützung für religiöse Glaubensvorstellungen bietet, indem sie von Prämissen ausgeht, die weder religiöse Glaubensvorstellungen sind, noch voraussetzen, gewann während der Aufklärung die Vorherrschaft und scheint ihre Wurzeln im Englischen Deismus des frühen achtzehnten Jahrhunderts zu haben. Sowohl Samuel Clarkes Demonstration of the Being and Attributes of God (1706) als auch George Cheynes Philosophical Principles of Religion, Natural and Revealed (1715) bereiteten den Weg für Gedankengänge, die man nun als klassische Formulierungen dessen sehen kann, was durch eine Folge von historischen Gegebenheiten die vorherrschende Verständnisweise von „Natürlicher Theologie“ geworden ist. Beide sind von Johann August Eberhard (1739-1809) in seiner wichtigen Vorlesung des Jahres 1781 „Vorbereitung zur natürlichen Theologie“ als bedeutende Quellen genannt worden, die Kants Darstellung dieses Themas beeinflusst hat und dadurch viel dazu beitrug, die Wahrnehmung in der deutschsprachigen Welt so zu prägen, dass dies als einzige Weise, dieses Konzept zu interpretieren, gilt. ${ }^{34}$ Doch diese rationalistische Natürliche Philosophie muss als eine spezielle Ausführung der Idee der natürlichen Theologie gesehen werden, die aus kulturellen Gründen die Vormachtstellung erlangt hat, und die historische Gegebenheiten des längst vergangenen „Zeitalters der Vernunft“ widerspiegelt. Barths Kritik der Natürlichen Theologie richtet sich demnach tatsächlich gegen diese spezielle Auslegung natürlicher Theologie. Wie diese Arbeit jedoch verdeutlicht hat, gibt es weitere Möglichkeiten, den Zweck und den Ort Natürlicher Theologie zu verstehen.

34 Vgl. Immanuel Kant, Gesammelte Schriften. 30 Bd. (Berlin: Reimer, 1902) Bd. 20, 491-606. Weiterhin vgl. Giovanna D'Aniello, "Von der Religion zur Theologie: Schleiermacher als Schüler Eberhards?," in Ein Antipode Kants? Johann August Eberhard im Spannungsfeld von spätaufklärerischer Philosophie und Theologie, Hrsg. Hans-Joachim Kertscher and Ernst Stöckmann (Berlin: De Gruyter, 2012) 165-84; Thomas HankE, "Kein Wunder und keine Instruktion: Kants Umgang mit dem Offenbarungsbegriff vor und in der Religionsschrift als Beitrag zu dessen diskreter Transformation," in Kant und die biblische Offenbarungsreligion, Hrsg. Jakub SIRovátKa and David VOP̌̌ADA (Prague: Karolinum, 2013), 16-27. 
Es besteht kein Zweifel, dass das vorherrschende Verständnis von Natürlicher Theologie eine Reaktion auf ein besonderes Zusammenspiel historischer Fakten darstellte. Natürliche Theologie verstanden als Mittel, um religiösen Glauben zu erlangen, ohne auf irgendwelche Instrumente kirchlicher Autorität zurückzugreifen, sondern durch Betrachtung der natürlichen Ordnung, erblühte in England im Laufe des siebzehnten Jahrhunderts, teils als Reaktion auf politische und intellektuelle Entwicklungen, die Unbehagen, gelegentlich Argwohn gegenüber traditionell christlicher Herangehensweisen an die Offenbarung hervorgerufen hatten. ${ }^{35}$

Mehrere Faktoren scheinen dieses neue Interesse an „Natürlicher Theologie“ (zu dieser Zeit in England oft als „Physikotheologie“ bezeichnet) beeinflusst zu haben. ${ }^{36}$ Wir können drei festhalten:

1. Das Aufkommen der Bibelkritik stellte die Verlässlichkeit oder Verständlichkeit der Schrift in Frage und schuf damit Interesse an den offenbarenden Kapazitäten der natürlichen Welt.

2. Die politischen Krisen der Zeit schufen ein wachsendes Misstrauen gegenüber den kirchlichen Autoritäten, was manche dazu veranlasste, neue Wissensquellen zu erforschen, die außerhalb kirchlicher Kontrolle gesehen wurden, wie eine Berufung auf die Vernunft oder die natürliche Ordnung.

3. Ein Misstrauen gegenüber organisierter Religion und christlichen Lehren veranlasste manche dazu, nach einer einfacheren „Religion der Natur“ zu suchen, in der die Natur als Quelle der Offenbarung geschätzt wurde.

Diese Entwicklungen können auf bestimmte Art als Bestätigung für die Bedenken gegenüber dem Weltbild des achtzehnten Jahrhunderts angesehen werden, nach Karl Barths berühmter Formulierung, dass es die menschliche Autonomie vor und gegen die göttliche Selbstoffenbarung stellte. ${ }^{37}$ „Natürliche Theologie“, wie Barth sie verstand, verkörpert die typische Tendenz der sündigen Menschheit, ihre epistemische und soteriologische Unabhängigkeit zu beweisen. Die Menschheit kann Gott unter selbst gewählten Bedingungen entdecken und eine Beziehung zu

35 Vgl. Scott Mandelbrote, “The Uses of Natural Theology in Seventeenth-Century England," Science in Context 20 (2007), 451-80.

36 Vgl. Richard S. WestFall, "The Scientific Revolution of the Seventeenth Century: A New World View," in The Concept of Nature, Hrsg. John Torrance (Oxford: Oxford University Press, 1992), 6393.

$37 \mathrm{Zu}$ Barths allgemeiner Einschätzung dieser Entwicklung empfiehlt sich sein wichtiger Aufsatz über den Menschen und die Menschlichkeit im achtzehnten Jahrhundert: Karl BARTH, Die protestantische Theologie im 19. Jahrhundert: Ihre Vorgeschichte und ihre Geschichte. 2. Aufl. (Zürich: Evangelischer Verlag, 1952), 16-59. Zur Bedeutung des Themas „Autonomie“ vgl. Christof GEsтRICH, Neuzeitliches Denken und die Spaltung der dialektischen Theologie: Zur Frage der natürlichen Theologie. (Tübingen: Mohr, 1977). 
ihm herstellen, statt unter jenen, die durch die christliche Verkündung vorgeben sind. Wenn das Wissen um Gott unabhängig von Gottes Selbstoffenbarung durch Christus erreicht werden kann, folgt daraus, dass die Menschheit den Ort, die Zeit und den Weg vorgeben kann, wie sie Gott erkennt. ${ }^{38}$

Diese bestimmte kulturelle Situation ist jedoch vorbei und mit ihrem Ende sind neue Arten entstanden, wie Natürliche Theologie verstanden und umgesetzt werden kann. Das traditionelle Verständnis dieses Begriffs aufrechtzuerhalten, hält uns in einer entfernten Vergangenheit gefangen und versperrt den Weg zu wichtigen theologischen Möglichkeiten. 1940 stellte Ludwig Wittgenstein fest: „Man muß manchmal einen Ausdruck aus der Sprache herausziehen, ihn zum Reinigen geben - und kann ihn dann wieder in den Verkehr einführen. " ${ }^{39}$ Können wir also den Begriff „Natürliche Theologie“ reinigen und wieder in den allgemeinen Gebrauch aufnehmen? Oder müssen wir uns seiner unvermeidlichen Bedeutungsvielfalt stellen und letztendlich damit leben lernen? Vielleicht können wir darauf hoffen, ein tiefer gehendes Verständnis der Idee zu entdecken, das uns zu begreifen hilft, warum sie auf so viele verschiedene Weisen verstanden wird, und dadurch eine innere Kohärenz ausmachen zu können, die dieser Pluralität zugrunde liegt.

Ich habe meine eigenen Ansichten zu diesen Themen. Der entscheidende kritische Punkt, den ich in diesem Artikel machen möchte, ist schlichtweg dieser: die Erneuerung der Natürlichen Theologie hängt von einer erneuten Definition ab, die es ermöglicht, dass ältere und alternative Ansätze - egal ob sie unter diesem Namen eingeordnet werden oder nicht - in einem Dialog mit dem theologischen Mainstream gesetzt werden können. Wir können es nicht zulassen, dass diese entscheidende kritische Debatte von wenig hilfreichen Annahmen über die genaue Identität und den Fokus der Natürlichen Theologie über die Maßen beeinflusst wird. In dieser Hinsicht ist eine kritische historische Erkundung befreiend, da sie uns dabei hilft, die Tiefe und Komplexität der Verständnisweisen - seien sie nun explizit oder implizit - der Natur und des Umfangs der Natürlichen Theologie innerhalb theologischer und philosophischer Überlegungen der christlichen Tradition zu begreifen. Nur dann können wir unseren Weg zurück zu einem vernünftigen Verständnis von Natürlicher Theologie finden, was für unsere Generation eine genauso dringende Aufgabe ist, wie sie es für die Brunners war.

$38 \mathrm{Zu}$ diesem Thema allgemein vgl. Regin Prenter, "Das Problem der natürlichen Theologie bei Karl Barth,” in Theologische Literaturzeitung 77 (1952), 607-11.

39 Ludwig Wittgenstein, Vermischte Bemerkungen/Culture and Value. (Oxford: Blackwell, 1998), 44. [Hervorhebung im Original]. 